\title{
Einige Bemerkungen zu der vorstehenden Arbeit des Herrn A. Speiser ${ }^{1}$ ).
}

Von

I. Schur in Berlin

Das Problem, die einfachsten algebraischen Zahlkörper zu bestimmen, in denen eine gegebene endliche Gruppe linearer Substitutionen rational darstellbar ist, gehört zu den schwierigsten Aufgaben der Gruppentheorie. In der vorstehenden Arbeit ist es Herrn Speiser gelungen, zu den bis jetzt erledigten Spezialfällen einige neue interessante Fälle hinzuzufügen. Besonders bemerkenswert ist sein Satz über irreduzible Gruppen ungeraden Grades mit reellem Charakter. Die Grundlage seiner Untersuchungen bildet der an und für sich interessante zahlentheoretische Satz, den Herr Speiser im $\S 1$ seiner Arbeit ableitet. Im folgenden will ich zeigen, daß die in meiner Arbeit ,Neue Begründung der Theorie der Gruppencharaktere “" $)$ entwickelten Methoden es gestatten, auf kürzerem Wege ein allgemeineres Resultat zù erhalten.

Es bedeute wie bei Herrn Speiser $K$ einen algebraischen Zahlkörper, der in b̧ezug auf den Grundkörper $k$ ein Normalkörper ist. Die Galo is sche Gruppe von $K$ relativ zu $k$ sei

$$
\mathscr{S}=G_{0}+G_{1}+\ldots+G_{g-1}
$$

Ist $A=\left(a_{x 2}\right)$ eine Matrix, deren Koeffizienten dem Körper $K$ angehören, so bezeichne man die zu $A$ in bezug auf $k$ konjugiert algebraischen Matrizen mit

$$
A^{S}=\left(a_{\varkappa \lambda}^{S}\right) \quad\left(S=G_{0}, G_{1}, \ldots, G_{g-1}\right) .
$$

Ordnet man den $g$ Elementen $S$ von $\left(S\right.$ in $K$ rationale Matrizen $M_{S}$ des Grades $m$ mit nicht verschwindenden Determinanten zu, für die Gleichungen der Form

1) Vgl. A. Speiser, Zahlentheoretische Sätze aus der Gruppentheorie. Diese Zeitschrift, 5 (1919), S. 1-6.

2) Sitzungsberichte der Berliner Akademie, Jahrg. 1905, S. 406-432. 


$$
M_{S}^{T} H_{T} \quad r_{S . T} M_{s T} \quad\left(S, T \quad G_{0}, G_{1} \ldots, G_{g-1}\right)
$$

bestehen, so sprechen wir von einer zum Faktorensystem $r_{s} x$ gehörenden Darstellung $\mathfrak{M} \quad\left\{M_{S}\right\}$ des Grades $m$ von (5). Für jede in $K$ rationale Matrix $\boldsymbol{A}$ des Grades $m$, deren Determinante nicht verschwindet, bilden zugleich mit den $M_{S}$ auch die Matrizen

$$
N_{S} \quad A^{S} M_{S} A^{-1}
$$

eine zum Faktorensystem $r_{s, T}$ gehörende Darstellung $\mathfrak{R}$, die zu $\mathfrak{M}$ äquivalent heißen möge. Die Darstellung $\mathfrak{M}$ nennen wir irreduzibel, wenn sich keine $z u$ ihr äquivalente Darstellung $\mathfrak{l}$ angeben läßt, die in den üblichen Bezeichungen die Form

hat.

$$
\mathfrak{R} \quad\left(\begin{array}{cc}
\mathfrak{J}_{1} & 0 \\
\mathfrak{U}_{3} & \mathfrak{M}_{1}
\end{array}\right)
$$

Multipliziert man die Matrizen $M_{S}$ der Darstellung $\mathfrak{M}$ mit irgend welchen von Null verschiedenen Größen $c_{S}$ des Körpers $K$, so bilden die Matrizen $c_{S} M_{S}$ eine neue Darstellung, die zum Faktorensystem

$$
r_{S, T}^{\prime}={ }_{c_{S T} c_{S, T}}^{c_{T}} r_{S,}
$$

gehört. Zwei derartige Faktorensysteme sind als nicht wesentlich von einander verschieden anzusehen, sie mögen als einander assoziiert bezeichnet werden. Gehört zu einem Faktorensystem $r_{r, s}$ eine Darstellung $\left\{M_{S}\right\}$ des Grades $m$, so lehrt die Betrachtung der Determinanten der Matrizen $M_{S}$, daß die $m$-ten Potenzen der Zahlen $r_{S, T}$ ein dem System $\varrho_{S, T}-1$ assoziiertes Faktorensystem bilden.

Satz I. Damit sich zu $g^{2}$ von $N u l l$ verschiedenen Größen $r_{S, T}$ des Körpers $K$ eine zu diesem Faktorensystem gehörende Darstellung der Cruppe (S) angeben lasse, ist notuendig und hinreichend, daß sie den $g^{3}$ Gleichungen

$$
r_{S, T}^{U} r_{S T, U}=r_{S, T U} r_{T, U} \quad\left(S, T, U=G_{0}, G_{1}, \ldots, G_{g-1}\right)
$$

genügen. In jedem Falle ist das Faktorensystem $r_{S, T}^{a}$ dem System $\varrho_{S, T}=1$ assoziiert.

Daß für die Größen $r_{S, x}$ eines Faktorensystems die Gleichungen (z) bestehen müssen, folgt unmittelbar aus den" Gleichungen (1) auf Grund des assoziativen Gesetzes. Andere Bedingungen kommen aber nicht hinzu. Denn genügen die (von Null verschiedenen) Größen $r_{S, T}$ von $K$ den Gleichungen (2) und setzt man $\varepsilon_{S}$ gleich 1 oder 0 , je nachdem $S$ dem Einheitselement $E$ von $\mathbb{G}$ gleich oder von ihm verschieden ist, so bilden, wie man leicht zeigt, die $g$ Matrizen 


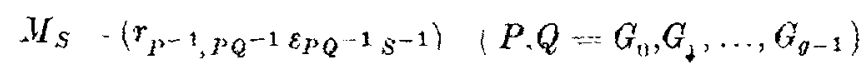

des Grades $g$ eme zum Faktorensystem $r_{S, T}$ gehörende Darstellung der Gruppe $\left(\mathfrak{S}^{3}\right)$. Hirraus folgt zugleich unsere Behauptung iber die Zahlen $r_{S . T}$.

Satz II. Sint $\mathbb{M}_{-}-\left\{M_{S}\right\}$ und $\mathfrak{R}-\left\{N_{S}\right\}$ zwei zum Fahtorensystem $r_{S . T}$ gehórende Darstellungen der Grade $m$ und $n \geq m$ von (\$3) so läßt sich stel eine Matrix $A$ des Ranges $m$ mit $m$ Zeilen und $n$ Spalten angeb' $n$, deren Koeffizienten dem Korper $K$ angehoren und die den $g$ Gleichungen

genügt.

$$
A^{s} N_{S} \quad H_{S} A \quad\left(S \quad G_{0}, G, \ldots, G_{g-1}\right)
$$

Ist nämlich $U$ eine beliebige in $K$ rationale Matrix mit $m$ Zeilen und $n$ Spalten, uird setzt man

$$
A=\sum_{R}^{\top} M_{n}^{-1} U^{R} N_{R}, \quad\left(R \quad G_{0}, G_{1} \ldots,\left(G_{y-1}\right)\right.
$$

so wird

$M_{S}^{-1} A^{S} N_{S}-\sum_{R} M_{S}^{-1}\left(M_{R}^{S}\right)^{-1} U^{R S} \cdot r_{R S} N_{R S} \cdots \sum_{n}^{\bigcup^{\top}} M_{R S}^{-1} U^{n s} N_{n S} \quad A$,

weil

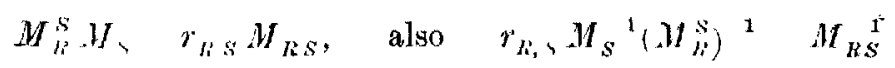

ist. Wir habon also nu noth zu zeigen, Aaß die Matrix (5) boi passender Wahl von $U^{\gamma}$ den Rang $m$ erhält. Dies rgibt sich folgendermaBen.

Bilden die Zahlen $\left(t_{1}, w_{2}, \ldots, \omega_{g}\right.$ eine Basis des Körpers $K$ in bezug auf den Grundkörper $k$, so kann $U \sum\left(0, U_{,}\right.$gesetzt werden, wobei die Koeffizienten $u_{n,{ }^{\prime}}^{(\gamma)}$ der $g$ Matrizen $U_{y}$ beliebige Größen des Körpers $k$ bedeuten können. Es wird dann

$$
U^{n}=\sum_{\gamma=1}^{a} \omega_{\gamma}^{n} U_{\gamma} .
$$

Man fasse nun irgendeine Unterdeterminante $D$ des Grades $m$ von $A$ ins Auge. Würde $D$ für alle $u_{t \neq \nu}^{(y)}$ von $k$ verschwinden, so müßte das auch zutreffen, wemn die $u_{k \neq}^{(y)}$ ganz beliebige Größen bedeuten. Da aber die Determinante der $g^{*}$ Zahlen $\omega_{y}^{R}$ von Null verschieden ist, so können wir die $U_{\gamma}$ so wählen, daß in ( 6 ) an Stelle der zu $U$ algebraisch konjugierten Matrizen $U^{k}$ beliebig vorgeschriebene Matrizen $U_{k}$ treten. Ist nun $B$ eine ganz beliebige Matrix mit $m$ Zeilen und $n$ Spalten und setzen wir $U_{R}=M_{R} B N_{R}^{-1}$, so tritt in (5) an Stelle von $A$ die Matrix $g B$. Da

3) Vgl. die analoge Betrachtung in meiner Arbeit, "Uber die Darstellung der endlichen Gruppen durch gebrochene lineare Substitutionen", Journ. für Math., 127 (1904), S. $20-50$;s. 24 ]. 
I. Schur. Bemerkungen zu der vorstehenden Arbeit des Herrn A Speiser.

hierin die Unterdeterminante $D$ gewiB nicht identisch Null ist, so kann $D$ auch nicht für alle Matrizen $U$ mit Koeffizienten aus dem Körper $K$ verschwinden.

Nimmt man insbesondere $m=n$ an, so folgt aus dem Satze II, daß zwei Darstellungen, die zu demselben Faktorensystem gehören, stets einander äquivalent sind, wenn nur ihre Grade übereinstimmen. Für das Faktorensystem $r_{S, T}=1$ liefert dies schon das Resultat des Herrn Speiser.

Ist ferner $m<n$ und genügt die in $K$ rationale Matrix $A$ des Ranges $m$ den Gleichungen (4), so können wir zwei Matrizen $P$ und $Q$ der Grade $m$ und $n$ mit nicht verschwindenden Determinanten bestimmen, deren Koeffizienten dem Körper $K$ angehören, so daß

$$
A^{*}=P A Q^{-1}=\left(E_{m}, 0\right)
$$

wird, wobei $E_{m}$ die Einheitsmatrix des Grades $m$ bedeutet. Setzt man dann

so wird

$$
P^{s} M_{S} P^{-1}=M_{S}^{*}, \quad Q^{s} N_{S} Q^{-1}=N_{S}^{*},
$$

$$
A^{*} N_{S}^{*}=M_{S}^{*} A^{*} \text {. }
$$

Hieraus folgt aber, daß die Darstellung $\mathfrak{R}^{*}=\left\{N_{S}^{*}\right\}$ die Form

$$
\mathfrak{R}^{*}=\left(\begin{array}{cc}
\mathfrak{M}^{*} & 0 \\
\mathfrak{R}_{3} & \mathfrak{\Re}_{4}
\end{array}\right)
$$

hat. Sind also insbesondere $\mathfrak{M}$ und $\mathfrak{R}$ beide irreduzibel, so muß $m=n$ sein und die beiden Darstellungen sind einander äquivalent. Als Schlußresultat ergibt sich nun ohne weiteres:

Satz III. Sieht man zwei äquivalente Darstellungen als nicht voneinander verschieden an, so gehört $z u$ jedem Faktorensystem $r_{S, i}$ nur eine irreduzible Darstellung $\mathfrak{M}$. Der Grad $n$ jeder anderen $z u \cdot r_{S, T}$ gehörenden Darstellung $\mathfrak{R}$ ist ein Vielfaches des Grades $m$ von $\mathfrak{M}$ und $\mathfrak{R}$ ist der Darstellung

$$
\left(\begin{array}{cccc}
\mathfrak{M} & 0 & 0 & \ldots \\
0 & \mathfrak{M} & 0 & \ldots \\
0 & 0 & \mathfrak{M} & \ldots \\
\ldots & \ldots & \ldots & \ldots
\end{array}\right)
$$

äquivalent, die $\mathfrak{M}$ genau $\frac{n}{m}-$ mal enthält. Diẹs gilt insbesondere auch für die Darstellung (3), und daher ist $m$ ein Teiler der Ordnung $g$ der Gruppe 8 S. 